\title{
A Pairing-Based DAA Scheme Further Reducing TPM Resources
}

\author{
Ernie Brickell \\ Intel Corporation \\ ernie.brickell@intel.com
}

\author{
Jiangtao Li \\ Intel Labs \\ jiangtao.li@intel.com
}

\begin{abstract}
Direct Anonymous Attestation (DAA) is an anonymous signature scheme designed for anonymous attestation of a Trusted Platform Module (TPM) while preserving the privacy of the device owner. Since TPM has limited bandwidth and computational capability, one interesting feature of DAA is to split the signer role between two entities: a TPM and a host platform where the TPM is attached. Recently, Chen proposed a new DAA scheme that is more efficient than previous DAA schemes. In this paper, we construct a new DAA scheme requiring even fewer TPM resources. Our DAA scheme is about 5 times more efficient than Chen's scheme for the TPM implementation using the Barreto-Naehrig curves. In addition, our scheme requires much smaller size of software code that needs to be implemented in the TPM. This makes our DAA scheme ideal for the TPM implementation. Our DAA scheme is efficient and provably secure in the random oracle model under the strong Diffie-Hellman assumption and the decisional Diffie-Hellman assumption.
\end{abstract}

\section{Introduction}

The concept and a concrete scheme of Direct Anonymous Attestation (DAA) were first introduced by Brickell, Camenisch, and Chen [5] for remote anonymous authentication of a Trusted Platform Module (TPM). The DAA scheme was adopted by the Trusted Computing Group (TCG) [22], an industry standardization body that aims to develop and promote an open industry standard for trusted computing hardware and software building blocks. The DAA scheme was standardized in the TCG TPM Specification Version 1.2 [21] and has recently been adopted by ISO/IEC as an international standard.

A DAA scheme involves three types of entities: an issuer, signers, and verifiers. The issuer is in charge of verifying the legitimation of signers and of issuing a membership credential to each signer. A signer can prove membership anonymously to a verifier by creating a DAA signature. The verifier can verify the membership of the signer from the DAA signature but he cannot learn the identity of the signer. DAA scheme can be seen as a special group signature scheme without the open feature, i.e., a DAA signature cannot be opened by anyone including the issuer to find out the identity of the signer.

One interesting feature of DAA is that the signer role of DAA is split between two entities: a TPM and a host where the TPM is attached. The TPM is the main signer but has limited bandwidth, computational capability, and storage. The host is a helper with more computational power but is less trusted. The TPM is the real signer and has the private signing key. The host helps the TPM to compute DAA signatures, but is not allowed to learn the private signing key or forge a DAA signature without the involvement from the TPM.

After DAA was first introduced, it has drawn a lot of attention from both industry and cryptographic community, e.g., in $[10,18,1,8,6,12,11]$, to list a few. The original DAA scheme [5] is based on the strong RSA assumption. Recently several groups of researchers have constructed pairing-based DAA schemes to achieve better efficiency. The first pairing-based DAA scheme was proposed by Ernie, Chen, and Li [6, 7]. Chen, Morrissey, and Smart improved the BCL-DAA scheme using asymmetric pairing [12,13]. These DAA schemes are based on the LRSW assumption [19].

Brickell and Li proposed an extension of DAA called Enhanced Privacy ID (EPID) [8] and presented a concrete EPID scheme based on the $q$-SDH assumption [9]. The EPID schemes focus on the revocation capabilities and treat the signer as a single entity instead of combination of a TPM and a host. Independently Chen and Feng proposed a DAA scheme [15] using $q$-SDH assumption. Recently, Chen builds a new DAA 
scheme [11] on top of the EPID scheme [9] by reducing the size of the private signing key. As compared in [11], $q$-SDH based DAA schemes [15, 9, 11] are more efficient than LRSW-based DAA schemes $[6,7,12,13]$, especially in the efficiency of the signature verification algorithm.

To the best of our knowledge, Chen's DAA scheme [11] is the most efficient DAA scheme and it requires least amount of TPM resources ${ }^{1}$. In this paper, we give a simple improvement to Chen's DAA scheme. Our DAA scheme is about 5 times more efficient for the TPM implementation using the Barreto-Naehrig curves [2]. In addition, our scheme requires much smaller size of software code that needs to be implemented in the TPM. This makes our DAA scheme ideal for the TPM implementation. More specifically, let $e: G_{1} \times G_{2} \rightarrow G_{T}$ be a bilinear map function. The DAA scheme in [11] requires two exponentiations in $G_{1}$ and one exponentiation in $G_{T}$. Whereas our DAA scheme in this paper requires only three exponentiations in $G_{1}$. Our improvement seems to be small, but has significant impact to TPM for the following two reasons:

- Usually operations in $G_{1}$ are more efficient than the operations in $G_{T}$. According to the arguments in [13], exponentiation in $G_{1}$ is about $1 / 4$ the cost of exponentiation in $G_{T}$ for symmetric pairing. For highly efficient curve choices such as Barreto-Naehrig curves [2] with 128-bit security, $G_{1}$ is an elliptic curve group over $\mathbb{F}_{q}$ while $G_{T}$ is a subgroup of $\mathbb{F}_{q^{12}}$. Exponentiation in $G_{1}$ is about 14 times more efficient than the one in $G_{T}$. Thus the computation needed for TPM in our scheme is about 5 times more efficient than the one in DAA scheme [11] using the Barreto-Naehrig curves.

- In our scheme TPM only requires to implement $G_{1}$ while the other DAA schemes $[13,11,15]$ require TPM to implement both $G_{1}$ and $G_{T}$. For small hardware devices such as TPM, more software code means larger firmware image, larger flash storage needed, and more software validation required. Note that if TPM has already implemented EC-DSA or other ECC primitives for other purposes, the additional software code needed for implementing our DAA scheme is very minimum.

Rest of this paper is organized as follows. We first review the formal specification and security requirements of DAA in Section 2. We then review the definition of pairing and related security assumptions in Section 3. We present our DAA scheme in Section 4 and give the security proof in Section 5. We compare our scheme with the existing DAA schemes in Section 6 and conclude our paper in Section 7.

\section{Review Security Model of DAA}

In this section, we review the specification and security model of DAA proposed in [7]. The security model in [7] is simpler than the original DAA definition [5] and easier to understand the security properties of DAA. There are four types of players in a DAA scheme: an issuer $\mathcal{I}$, a TPM $\mathcal{M}_{i}$, a host $\mathcal{H}_{i}$ and a verifier $\mathcal{V}_{j} . \mathcal{M}_{i}$ and $\mathcal{H}_{i}$ form a platform in the trusted computing environment and share the role of a DAA signer. A DAA scheme has three polynomial-algorithms (Setup, Verify, Link) and two interactive protocols (Join, Sign):

Setup : On input of a security parameter $1^{k}, \mathcal{I}$ uses this randomized algorithm to produce a pair (gpk, isk), where isk is the issuer's secret key, and gpk is the public key including the global public parameters.

Join : This randomized algorithm consists of two sub-algorithms $\operatorname{Join}_{\mathrm{t}}$ and $\operatorname{Join}_{\mathrm{i}} . \mathcal{M}_{i}$ uses $\operatorname{Join}_{\mathrm{t}}$ to produce a pair $\left(\mathbf{s k}_{i}, \mathbf{c o m m}_{i}\right)$, where $\mathbf{s k}_{i}$ is the TPM's secret key and $\mathbf{c o m m}_{i}$ is a commitment of $\mathbf{s k}_{i}$. On input of $\mathrm{comm}_{i}$ and isk, $\mathcal{I}$ uses $\operatorname{Join}_{\mathrm{i}}$ to produce $\mathrm{cre}_{i}$, which is a DAA credential associated with $\mathrm{sk}_{i}$. Note that the value $\mathrm{cre}_{i}$ is given to both $\mathcal{M}_{i}$ and $\mathcal{H}_{i}$, but the value $\mathrm{sk}_{i}$ is known to $\mathcal{M}_{i}$ only.

Sign : On input of $\mathrm{sk}_{i}, \mathrm{cre}_{i}$, a basename $\mathrm{bsn}_{j}$ (the name string of $\mathcal{V}_{j}$ or a special symbol $\perp$ ), and a message $m$ that includes the data to be signed and the verifier's nonce $n_{V}$ for freshness, $\mathcal{M}_{i}$ and $\mathcal{H}_{i}$ use this randomized algorithm to produce a signature $\sigma$ on $m$ under $\left(\mathbf{s k}_{i}, \mathrm{cre}_{i}\right)$ associated with $\mathrm{bsn}_{j}$. The basename $\mathrm{bsn}_{j}$ is used for controlling the linkability.

Verify : On input of $m, \mathrm{bsn}_{j}$, a candidate signature $\sigma$ for $m$, and a set of revoked secret keys RL, $\mathcal{V}_{j}$ uses this deterministic algorithm to return either 1 (accept) or 0 (reject). How to build the revocation list is out the scope of the DAA scheme.

\footnotetext{
${ }^{1}$ The original CMS-DAA scheme [12] requires lesser TPM resources. However, there was a security flaw in their DAA scheme. The patched version [13] has the same computational complexity for TPM as in Chen's DAA scheme [11].
} 
Link: On input of two signatures $\sigma_{0}$ and $\sigma_{1}, \mathcal{V}_{j}$ uses this deterministic algorithm to return 1 (linked), 0 (unlinked) or $\perp$ (invalid signatures). Link will output $\perp$ if, by using an empty RL, either Verify $\left(\sigma_{0}\right)=0$ or Verify $\left(\sigma_{1}\right)=0$ holds. Otherwise, Link will output 1 if signatures can be linked or 0 if the signatures cannot be linked.

A DAA scheme is secure if it is correct, user-controlled-anonymous, and user-controlled-traceable.

Correctness If both the signer and verifier are honest, that implies $\mathbf{s k}_{i} \notin \mathrm{RL}$, the signatures and their links generated by the signer will be accepted by the verifier with overwhelming probability. This means that the DAA scheme must meet the following consistency requirement.

$(\mathrm{gpk}$, isk $) \leftarrow \operatorname{Setup}\left(1^{k}\right),\left(\mathrm{sk}_{i}, \operatorname{cre}_{i}\right) \leftarrow \operatorname{Join}(\mathrm{isk}, \mathrm{gpk}),\left.\left(m_{b}, \sigma_{b}\right) \leftarrow \operatorname{Sign}\left(m_{b}, \mathrm{bsn}_{j}, \mathrm{sk}_{i}, \mathrm{cre}_{i}, \mathrm{gpk}\right)\right|_{b=\{0,1\}}$, $\left.\left.\Longrightarrow 1 \leftarrow \operatorname{Verify}\left(m_{b}, \mathrm{bsn}_{j}, \sigma_{b}, \mathrm{gpk}, \mathrm{RL}\right)\right|_{b=\{0,1\}} \wedge 1 \leftarrow \operatorname{Link}\left(\sigma_{0}, \sigma_{1}, \mathrm{gpk}\right)\right|_{\mathrm{bsn}_{j} \neq \perp}$.

User-Controlled-Anonymity A DAA scheme is user-controlled-anonymous if no probabilistic polynomialtime adversary can win the following game between a challenger $\mathcal{C}$ and an adversary $\mathcal{A}$ as follows:

- Initial: $\mathcal{C}$ runs $\operatorname{Setup}\left(1^{k}\right)$ and gives the resulting isk and gpk to $\mathcal{A}$.

- Phase 1: $\mathcal{C}$ is probed by $\mathcal{A}$ who makes the following queries:

- Sign. $\mathcal{A}$ submits a signer's identity $S$, a basename bsn (either $\perp$ or a data string) and a message $m$ of his choice to $\mathcal{C}$, who runs Sign to get a signature $\sigma$ and responds with $\sigma$.

- Join. $\mathcal{A}$ submits a signer's identity $S$ of his choice to $\mathcal{C}$, who runs $\operatorname{Join}_{\mathrm{t}}$ with $\mathcal{A}$ to create sk and to obtain cre from $\mathcal{A}$. $\mathcal{C}$ verifies the validation of cre and keeps sk secret.

- Corrupt. $\mathcal{A}$ submits a signer's identity $S$ of his choice to $\mathcal{C}$, who responds with the value sk of the signer.

- Challenge: At the end of Phase $1, \mathcal{A}$ chooses two signers' identities $S_{0}$ and $S_{1}$, a message $m$ and a basename bsn of his choice to $\mathcal{C}$. $\mathcal{A}$ must not have made any Corrupt query on either $S_{0}$ or $S_{1}$, and not have made the Sign query with the same bsn if bsn $\neq \perp$ with either $S_{0}$ or $S_{1}$. To make the challenge, $\mathcal{C}$ chooses a bit $b$ uniformly at random, signs $m$ associated with bsn under $\left(\mathbf{s k}_{b}, \mathrm{cre}_{b}\right)$ to get a signature $\sigma$ and returns $\sigma$ to $\mathcal{A}$.

- Phase 2: $\mathcal{A}$ continues to probe $\mathcal{C}$ with the same type of queries that it made in Phase 1 . Again, it is not allowed to corrupt any signer with the identity either $S_{0}$ or $S_{1}$, and not allowed to make any Sign query with bsn if bsn $\neq \perp$ with either $S_{0}$ or $S_{1}$.

- Response: $\mathcal{A}$ returns a bit $b^{\prime}$. We say that the adversary wins the game if $b=b^{\prime}$.

Definition 1 Let $\mathcal{A}$ denote an adversary that plays the game above. We denote by $\operatorname{Adv}\left[\mathcal{A}_{\mathcal{D} \mathcal{A} \mathcal{A}}^{\text {anon }}\right]=\mid \operatorname{Pr}\left[b^{\prime}=\right.$ $b]-1 / 2 \mid$ the advantage of $\mathcal{A}$ in breaking the user-controlled-anonymity game. We say that a DAA scheme is user-controlled-anonymous if for any probabilistic polynomial-time adversary $\mathcal{A}, \operatorname{Adv}\left[\mathcal{A}_{\mathcal{D} \mathcal{A} \mathcal{A}}^{\text {anon }}\right]$ is negligible.

User-Controlled-Traceability A DAA scheme is user-controlled-traceable if no probabilistic polynomialtime adversary can win the following game between a challenger $\mathcal{C}$ and an adversary $\mathcal{A}$ as follows:

- Initial: $\mathcal{C}$ executes Setup $\left(1^{k}\right)$ and gives the resulting gpk to $\mathcal{A}$. It keeps isk secret.

- Probing: $\mathcal{C}$ is probed by $\mathcal{A}$ who makes the following queries:

- Sign. The same as in the game of user-controlled-anonymity.

- Semi-sign. $\mathcal{A}$ submits a signer's identity $S$ along with the data transmitted from $\mathcal{H}_{i}$ to $\mathcal{M}_{i}$ in Sign of his choice to $\mathcal{C}$, who acts as $\mathcal{M}_{i}$ in Sign and responds with the data transmitted from $\mathcal{M}_{i}$ to $\mathcal{H}_{i}$ in the Sign protocol. 
- Join. There are two cases of this query. Case 1: $\mathcal{A}$ submits a signer's identity $S$ of his choice to $\mathcal{C}$, who runs Join to create sk and cre for the signer. Case 2: $\mathcal{A}$ submits a signer's identity $S$ with a sk value of his choice to $\mathcal{C}$, who runs Join $n_{i}$ to create cre for the signer and puts the given sk into RL. $\mathcal{C}$ responds the query with cre. Suppose that $\mathcal{A}$ does not use a single $S$ for both of the cases.

- Corrupt. This is the same as in the game of user-controlled-anonymity, except that at the end $\mathcal{C}$ puts the revealed sk into the list of RL.

- Forge: $\mathcal{A}$ returns a signer's identity $S$, a signature $\sigma$, its signed message $m$ and the associated basename bsn. We say that the adversary wins the game if

1. $\operatorname{Verify}(m, \mathrm{bsn}, \sigma, \mathrm{gpk}, \mathrm{RL})=1$ (accepted), but $\sigma$ is neither a response of the existing Sign queries nor a response of the existing Semi-sign queries (partially); and/or

2. In the case of $\mathrm{bsn} \neq \perp$, there exists another signature $\sigma^{\prime}$ associated with the same identity and bsn, and the output of $\operatorname{Link}\left(\sigma, \sigma^{\prime}\right)$ is 0 (unlinked).

Definition 2 Let $\mathcal{A}$ be an adversary that plays the game above. Let $\operatorname{Adv}\left[\mathcal{A}_{\mathcal{D} A \mathcal{A}}^{\text {trace }}\right]=\operatorname{Pr}[\mathcal{A}$ wins $]$ denote the advantage that $\mathcal{A}$ breaks the user-controlled-traceability game. We say that a DAA scheme is user-controlledtraceable if for any probabilistic polynomial-time adversary $\mathcal{A}, \operatorname{Adv}\left[\mathcal{A}_{\mathcal{D} \mathcal{A} \mathcal{A}}^{\text {trace }}\right]$ is negligible.

\section{Pairings and Complexity Assumptions}

\subsection{Background on Bilinear Maps}

Our DAA scheme use bilinear maps as a fundamental building block. We follow the notation of Boneh, Boyen, and Shacham [4] to review some background on pairings. Let $G_{1}$ and $G_{2}$ to two multiplicative cyclic groups of prime order $p$. Let $g_{1}$ be a generator of $G_{1}$ and $g_{2}$ be a generator of $G_{2}$. We say $e: G_{1} \times G_{2} \rightarrow G_{T}$ is an admissible bilinear map, if it satisfies the following properties:

1. Bilinear. For all $u \in G_{1}, v \in G_{2}$, and for all $a, b \in \mathbb{Z}, e\left(u^{a}, v^{b}\right)=e(u, v)^{a b}$.

2. Non-degenerate. $e\left(g_{1}, g_{2}\right) \neq 1$ and is a generator of $G_{T}$.

3. Computable. There exists an efficient algorithm for computing $e(u, v)$ for any $u \in G_{1}, v \in G_{2}$.

We call the two groups $\left(G_{1}, G_{2}\right)$ in the above a bilinear group pair. In the rest of this paper, we consider bilinear maps $e: G_{1} \times G_{2} \rightarrow G_{T}$ where $G_{1}, G_{2}$, and $G_{T}$ are multiplicative groups of prime order $p$.

\subsection{Strong Diffie-Hellman Assumption}

The security of our DAA scheme is related to the hardness of the q-SDH problem introduced by Boneh and Boyen [3]. Let $G_{1}$ and $G_{2}$ be two cyclic groups of prime order $p$, respectively, generated by $g_{1}$ and $g_{2}$. The $q$-Strong Diffie-Hellman $\left(q\right.$-SDH) problem in $\left(G_{1}, G_{2}\right)$ is defined as follows: Given a $(q+3)$-tuple of elements $\left(g_{1}, g_{1}^{\gamma}, \ldots, g_{1}^{\left(\gamma^{q}\right)}, g_{2}, g_{2}^{\gamma}\right)$ as input, output a pair $\left(g_{1}^{1 /(\gamma+x)}, x\right)$ where $x \in \mathbb{Z}_{p}^{*}$. An algorithm $\mathcal{A}$ has advantage $\epsilon$ in solving $q$-SDH problem in $\left(G_{1}, G_{2}\right)$ if

$$
\operatorname{Pr}\left[\mathcal{A}\left(g_{1}, g_{1}^{\gamma}, \ldots, g_{1}^{\left(\gamma^{q}\right)}, g_{2}, g_{2}^{\gamma}\right)=\left(g_{1}^{1 /(\gamma+x)}, x\right)\right] \geq \epsilon
$$

where the probability is over the random choice of $\gamma$ and the random bits of $\mathcal{A}$.

Definition 3 We say that the $(q, t, \epsilon)$-SDH assumption holds in $\left(G_{1}, G_{2}\right)$ if no $t$-time algorithm has advantage at least $\epsilon$ in solving the $q$-SDH problem.

The $q$-SDH assumption was used by Boneh and Boyen [3] to construct a short signature scheme without random oracles and was shown in the same paper that $q-\mathrm{SDH}$ assumption holds in the generic group in the sense of Shoup [20]. The $q$-SDH assumption was later used in [4] for constructing a short group signature scheme. The security of the SDH problem was studied by Cheon [16]. 


\subsection{Decisional Diffie-Hellman Assumption}

Let $G$, generated by $g$, be a cyclic group of prime order $p$. The Decisional Diffie-Hellman (DDH) problem in $G$ is defined as follows: Given a tuple of elements $\left(g, g^{a}, g^{b}, g^{c}\right)$ as input, output 1 if $c=a b$ and 0 otherwise. An algorithm $\mathcal{A}$ has advantage $\epsilon$ in solving DDH problem in $G$ if

$$
\left|\operatorname{Pr}\left[g \leftarrow G, a, b \leftarrow \mathbb{Z}_{p}: \mathcal{A}\left(g, g^{a}, g^{b}, g^{a b}\right)=1\right]-\operatorname{Pr}\left[g \leftarrow G, a, b, c \leftarrow \mathbb{Z}_{p}: \mathcal{A}\left(g, g^{a}, g^{b}, g^{c}\right)=1\right]\right| \geq \epsilon
$$

where the probability is over the random choice of the parameters to $\mathcal{A}$ and over the random bits of $\mathcal{A}$.

Definition 4 We say that the $(t, \epsilon)$-DDH assumption holds in $G$ if no $t$-time algorithm has advantage at least $\epsilon$ in solving the DDH problem in $G$.

Let $\left(G_{1}, G_{2}\right)$ be a bilinear group pair. Our DAA scheme requires the DDH problem for $G_{1}$ to be hard. The DDH assumption on $G_{1}$ is often known as the External Diffie-Hellman (XDH) assumption. This assumption is also used in Chen's DAA scheme [11].

\section{The Proposed DAA Scheme}

In this section, we present our construction of DAA scheme from bilinear maps. Our construction builds on top of the recent pairing-based EPID scheme [9] and Chen's DAA scheme [11]. The DAA scheme has three algorithms Setup, Verify, Link and two interactive protocols Join and Sign which are defined as follows.

\subsection{Setup Algorithm}

The setup algorithm is exactly the same as the one in [11]. On input of the security parameters $1^{t}$, the setup algorithm takes the following steps:

1. Choose an asymmetric bilinear group pair $\left(G_{1}, G_{2}\right)$ of prime order $p$ and a pairing function $e: G_{1} \times G_{2} \rightarrow$ $G_{T}$. Let $g_{1}$ and $g_{2}$ be the generators of $G_{1}$ and $G_{2}$, respectively.

2. Choose $h_{1}, h_{2} \leftarrow G_{1}, \gamma \leftarrow \mathbb{Z}_{p}^{*}$, and compute $w:=g_{2}^{\gamma}$.

3. Select five hash functions $H_{1}:\{0,1\}^{*} \rightarrow \mathbb{Z}_{p}, H_{2}:\{0,1\}^{*} \rightarrow \mathbb{Z}_{p}, H_{3}:\{0,1\}^{*} \rightarrow G_{1}, H_{4}:\{0,1\}^{*} \rightarrow \mathbb{Z}_{p}$, $H_{5}:\{0,1\}^{*} \rightarrow \mathbb{Z}_{p}$.

4. Compute $T_{1}=e\left(g_{1}, g_{2}\right), T_{2}=e\left(h_{1}, g_{2}\right), T_{3}=e\left(h_{2}, g_{2}\right)$, and $T_{4}=e\left(h_{2}, w\right)$.

5. Output the DAA public key and the issuer's private key

$$
\text { (gpk, isk) :=((G, } \left.\left.G_{2}, G_{T}, p, e, g_{1}, h_{1}, h_{2}, g_{2}, w, H_{1}, H_{2}, H_{3}, H_{4}, H_{5}, T_{1}, T_{2}, T_{3}, T_{4}\right), \gamma\right)
$$

Note that $T_{1}, T_{2}, T_{3}$, and $T_{4}$ are optional in gpk, as they can be computed from $g_{1}, h_{1}, h_{2}, g_{2}, w$ by the signers and verifiers. Also note that, in the actual implementation, we can choose the same hash function for $H_{1}, H_{2}, H_{4}$, and $H_{5}$. We use different hash functions in order to prove the security.

\subsection{Join Protocol}

The join protocol is the same as in [11] as well. This protocol is performed by a TPM $\mathcal{M}$, the corresponding host $\mathcal{H}$, and an issuer $\mathcal{I}$. Assume $\mathcal{M}$ and $\mathcal{I}$ have already established a secure authenticated channel using $\mathcal{M}$ 's endorsement key [21]. Let DAAseed be $\mathcal{M}$ 's internal secret seed. Let $K_{I}$ be $\mathcal{I}$ 's long term public key. In the join protocol, $\mathcal{M}$ chooses a unique secret key $\mathrm{sk}=f$ and then obtains a credential cre $=(A, x)$ from $\mathcal{I}$ such that $A=\left(g_{1} \cdot h_{1}^{f}\right)^{1 /(x+\gamma)}$. The join protocol takes the following steps.

1. $\mathcal{I}$ chooses a nonce $n_{I} \in\{0,1\}^{t}$ and sends $n_{I}$ as a challenge to $\mathcal{M}$.

2. $\mathcal{M}$ computes $f:=H_{1}\left(\right.$ DAAseed $\|$ cnt $\left.\| K_{I}\right)$, where cnt is a count value. $\mathcal{M}$ sets its secret key sk $:=f$. The purpose of using $K_{I}$ and cnt can be found in the original DAA scheme [5]. 
3. $\mathcal{M}$ chooses at random $r_{f} \leftarrow \mathbb{Z}_{p}$ and computes $F:=h_{1}^{f}$ and $R:=h_{1}^{r_{f}}$.

4. $\mathcal{M}$ computes $c:=H_{2}\left(\mathrm{gpk}\left\|n_{I}\right\| F \| R\right)$ and $s_{f}:=r_{f}+c \cdot f(\bmod p)$.

5. $\mathcal{M}$ sets comm $:=\left(F, c, s_{f}, n_{I}\right)$ and sends comm to $\mathcal{I}$.

6. $\mathcal{I}$ verifies the value of $n_{I}$ and checks $F$ against the revocation list.

7. I computes $\hat{R}:=h_{1}^{s_{f}} \cdot F^{-c}$ and verifies that $c=H_{2}\left(\mathrm{gpk}\left\|n_{I}\right\| F \| \hat{R}\right)$. If verification fails, then abort.

8. $\mathcal{I}$ chooses at random $x \leftarrow \mathbb{Z}_{p}$ and computes $A:=\left(g_{1} \cdot F\right)^{1 /(x+\gamma)}$.

9. $\mathcal{I}$ sets the DAA credential cre $:=(A, x)$ and sends cre to $\mathcal{M}$.

10. $\mathcal{M}$ forwards $F$ and cre to $\mathcal{H}$.

11. $\mathcal{H}$ verifies that $e\left(A, w g_{2}^{x}\right)=e\left(g_{1} F, g_{2}\right)$. If verification fails, then abort.

Note that the TPM $\mathcal{M}$ and the host $\mathcal{H}$ have a DAA signing key $(A, x, f)$ such that $e\left(A, w g_{2}^{x}\right)=e\left(g_{1} h_{1}^{f}, g_{2}\right)$. In the DAA schemes $[9,15]$, the signing key is $(A, x, y, f)$ such that $e\left(A, w g_{2}^{x}\right)=e\left(g_{1} h_{1}^{f} h_{2}^{y}, g_{2}\right)$. Therefore our scheme has a smaller signing key.

\subsection{Sign Protocol}

This join protocol is performed by a TPM $\mathcal{M}$ and a host $\mathcal{H}$, where $\mathcal{M}$ has the secret key $f$ and $\mathcal{H}$ has the credential $(A, x)$. The other input of the protocol is the DAA public key gpk, a message $m$ to be signed, and a basename bsn and a nonce $n_{V}$ from the verifier. In this protocol, the signer chooses $B \in G_{1}$ and computes $K:=B^{f}$, then uses zero-knowledge proof to prove

$$
P K\left\{(A, x, f): e\left(A, w g_{2}^{x}\right)=e\left(g_{1} h_{1}^{f}, g_{2}\right) \wedge K=B^{f}\right\}
$$

As in most of DAA schemes, the $(B, K)$ pair is used for revocation check. To prove $e\left(A, w g_{2}^{x}\right)=e\left(g_{1} h_{1}^{f}, g_{2}\right)$ holds, the signer first computes $T=A \cdot h_{2}^{a}$ where $a$ is randomly chosen, then proves the following equation

$$
e\left(T, g_{2}\right)^{-x} \cdot e\left(h_{1}, g_{2}\right)^{f} \cdot e\left(h_{2}, g_{2}\right)^{a x} \cdot e\left(h_{2}, w\right)^{a}=e(T, w) / e\left(g_{1}, g_{2}\right) .
$$

The overall approach here is the same as in [11]. The main difference between our scheme and Chen's DAA scheme [11] is on how we divide the computation between $\mathcal{M}$ and $\mathcal{H}$ in a secure way. The join protocol takes the following steps:

1. If bsn $=\perp, \mathcal{M}$ chooses $B \leftarrow G_{1}$, otherwise, $\mathcal{M}$ computes $B:=H_{3}(\mathrm{bsn})$.

2. $\mathcal{M}$ chooses at random $r_{f} \leftarrow \mathbb{Z}_{p}$ and computes

$$
K:=B^{f}, \quad R_{1}:=B^{r_{f}}, \quad R_{2 t}:=h_{1}^{r_{f}} .
$$

3. $\mathcal{M}$ sends $\left(B, K, R_{1}, R_{2 t}\right)$ to $\mathcal{H}$.

4. $\mathcal{H}$ chooses $a \leftarrow \mathbb{Z}_{p}$, computes $b:=a \cdot x(\bmod p)$, and $T:=A \cdot h_{2}^{a}$.

5. $\mathcal{H}$ randomly picks

$$
r_{x} \leftarrow \mathbb{Z}_{p}, \quad r_{a} \leftarrow \mathbb{Z}_{p}, \quad r_{b} \leftarrow \mathbb{Z}_{p} .
$$

6. $\mathcal{H}$ computes

$$
\begin{aligned}
R_{2} & :=e\left(T, g_{2}\right)^{-r_{x}} \cdot e\left(h_{1}, g_{2}\right)^{r_{f}} \cdot e\left(h_{2}, g_{2}\right)^{r_{b}} \cdot e\left(h_{2}, w\right)^{r_{a}}, \\
& :=e\left(R_{2 t} \cdot T^{-r_{x}} \cdot h_{2}^{r_{b}}, g_{2}\right) \cdot T_{4}^{r_{a}} .
\end{aligned}
$$


7. $\mathcal{H}$ computes $c_{h}:=H_{4}\left(\operatorname{gpk}\|B\| K\|T\| R_{1}\left\|R_{2}\right\| n_{V}\right)$ and sends $c_{h}$ to $\mathcal{M}$.

8. $\mathcal{M}$ chooses a random nonce $n_{T} \leftarrow\{0,1\}^{t}$ and computes $c:=H_{5}\left(c_{h}\left\|n_{T}\right\| m\right)$.

9. $\mathcal{M}$ computes in $s_{f}:=r_{f}+c \cdot f(\bmod p)$.

10. $\mathcal{M}$ sends $\left(c, n_{T}, s_{f}\right)$ to $\mathcal{H}$. $\mathcal{M}$ erases $r_{f}$ after sending this message.

11. $\mathcal{H}$ computes

$$
s_{x}:=r_{x}+c \cdot x(\bmod p), \quad s_{a}:=r_{a}+c \cdot a(\bmod p), \quad s_{b}:=r_{b}+c \cdot b(\bmod p) .
$$

12. $\mathcal{H}$ outputs $\sigma:=\left(B, K, T, c, n_{T}, s_{f}, s_{x}, s_{a}, s_{b}\right)$.

Note that the signing protocol is a three-message protocol: In the first message, $\mathcal{M}$ sends $\left(B, K, R_{1}, R_{2 t}\right)$ to $\mathcal{H}$. In the second message, $\mathcal{H}$ sends $c_{h}$ to $\mathcal{M}$. In the third message, $\mathcal{M}$ sends $\left(c, n_{T}, s_{f}\right)$ to $\mathcal{H}$. The way we divide the computation between the TPM and the host is similar to the one in the original DAA paper [5].

As pointed out by Chen et al. [13] recently, such signing protocols are vulnerable to "replay" attack. That is, if a malicious host engages with a TPM on the signing protocol by submitting a $c_{h}$ value and obtains $\left(c, n_{T}, s_{f}\right)$ from the TPM. The host then re-submits $c_{h}$ again and somehow tricks the TPM to start from step 8 of the protocol, then the TPM will output a different set $\left(c^{\prime}, n_{T}^{\prime}, s_{f}^{\prime}\right)$. The host can figure the private key $f$ from $\left(c, c^{\prime}, s_{f}, s_{f}^{\prime}\right)$. In fact, this type of vulnerability applies to any interactive zero-knowledge proof protocol. If attacker can replay the challenge, then he can extract the prover's knowledge. Such vulnerability can be easily mitigated using stage control mechanism. For example, if the TPM receives the second message $c_{h}$ from the host, it checks whether it has a pending signing protocol. If not, it simply rejects $c_{h}$. In our scheme, we use an even simpler method: the TPM deletes $r_{f}$ after sending $s_{f}$ to the host. This can prevent the replay attack.

\subsection{Verify Algorithm}

On input of a message $m$, a basename bsn, a nonce $n_{V}$, a signature $\left(B, K, T, c, n_{T}, s_{f}, s_{x}, s_{a}, s_{b}\right)$, the public key gpk, and the revocation list RL (a list of revoked secret keys), the verification algorithm takes the following steps:

1. Verify that $B, K, T \in G_{3}$ and $s_{f}, s_{x}, s_{a}, s_{b} \in \mathbb{Z}_{p}$.

2. Compute $\hat{R}_{1}:=B^{s_{f}} \cdot K^{-c}$.

3. Compute

$$
\begin{aligned}
\hat{R}_{2} & :=e\left(T, g_{2}\right)^{-s_{x}} \cdot e\left(h_{1}, g_{2}\right)^{s_{f}} \cdot e\left(h_{2}, g_{2}\right)^{s_{b}} \cdot e\left(h_{2}, w\right)^{s_{a}} \cdot\left(e\left(g_{1}, g_{2}\right) / e(T, w)\right)^{c} . \\
& :=e\left(T, g_{2}^{-s_{x}} \cdot w^{-c}\right) \cdot T_{1}^{c} \cdot T_{2}^{s_{f}} \cdot T_{3}^{s_{b}} \cdot T_{4}^{s_{a}}
\end{aligned}
$$

4. Verify that

$$
c \stackrel{?}{=} H_{5}\left(H_{4}\left(\operatorname{gpk}\|B\| K\|T\| \hat{R}_{1}\left\|\hat{R}_{2}\right\| n_{V}\right)\left\|n_{T}\right\| m\right) .
$$

5. For each $f^{\prime} \in \mathrm{RL}$, if $K=B^{f^{\prime}}$, output 0 (reject).

6. If any of the above verifications fails, output 0 (reject), otherwise, output 1 (accept).

\subsection{Link Algorithm}

On input of two message-signature pairs $\left(m_{0}, \sigma_{0}\right)$ and $\left(m_{1}, \sigma_{1}\right)$, a basename bsn, and the public key gpk, the link algorithm performs the following steps:

1. For each signature $\sigma_{b}$ where $b \in\{0,1\}$, run the verify algorithm Verify $\left(\sigma_{b}, m_{b}\right.$, bsn, gpk). If either of two verifications returns 0 (reject), output $\perp$.

2. If $(B, K) \in \sigma_{0}$ are the same as $(B, K) \in \sigma_{1}$, return 1 (linked), otherwise return 0 (unlinked). 


\section{Security Proof}

In this section, we prove our DAA scheme is secure under the security definitions stated in Section 2 . We show that our DAA scheme is correct, user-controlled-anonymous, and user-controlled-traceable. The security of the DAA scheme based on the $q-\mathrm{SDH}$ assumption and $G_{1}-\mathrm{DDH}$ assumption defined in Section 3.

Theorem 1 The DAA scheme in Section 4 is correct.

Proof. To show the DAA scheme is correct, we prove that a signature created by a valid and unrevoked signer can be successfully verified by any verifier. In order to have a success signature verification, $\hat{R}_{1}, \hat{R}_{2}$ in the verify algorithm must be equal to $R_{1}, R_{2}$ in the sign protocol, respectively. We prove $\hat{R}_{1}=R_{1}$ and $\hat{R}_{2}=R_{2}$ as follows.

$$
\begin{aligned}
\hat{R}_{1} & =B^{s_{f}} \cdot K^{-c}=B^{r_{f}} \cdot B^{c f} \cdot\left(B^{f}\right)^{-c}=B^{r_{f}}=R_{1} \\
\hat{R}_{2} & =e\left(T, g_{2}\right)^{-s_{x}} \cdot e\left(h_{1}, g_{2}\right)^{s_{f}} \cdot e\left(h_{2}, g_{2}\right)^{s_{b}} \cdot e\left(h_{2}, w\right)^{s_{a}} \cdot\left(e\left(g_{1}, g_{2}\right) / e(T, w)\right)^{c} \\
& =R_{2} \cdot e\left(T, g_{2}\right)^{-c x} \cdot e\left(h_{1}, g_{2}\right)^{c f} \cdot e\left(h_{2}, g_{2}\right)^{c b} \cdot e\left(h_{2}, w\right)^{c a} \cdot\left(e\left(g_{1}, g_{2}\right) / e(T, w)\right)^{c} \\
& =R_{2} \cdot\left(e\left(g_{1}, g_{2}\right) \cdot e\left(h_{1}, g_{2}\right)^{f} \cdot e\left(h_{2}, g_{2}\right)^{b} \cdot e\left(h_{2}, w\right)^{a} \cdot e\left(T, g_{2}\right)^{-x} \cdot e(T, w)^{-1}\right)^{c} \\
& =R_{2} \cdot\left(e\left(g_{1}, g_{2}\right) \cdot e\left(h_{1}, g_{2}\right)^{f} \cdot e\left(h_{2}, g_{2}\right)^{a x} \cdot e\left(h_{2}, w\right)^{a} \cdot e\left(T, g_{2}^{x} w\right)^{-1}\right)^{c} \\
& =R_{2} \cdot\left(e\left(g_{1} h_{1}^{f}, g_{2}\right) \cdot e\left(h_{2}^{a}, g_{2}^{x} w\right) \cdot e\left(A, g_{2}^{x} w\right)^{-1} \cdot e\left(h_{2}^{a}, g_{2}^{x} w\right)^{-1}\right)^{c}=R_{2}
\end{aligned}
$$

The last equation holds because for a valid private key $(A, x, f), e\left(A, g_{2}^{x} w\right)=e\left(g_{1} h_{1}^{f}, g_{2}\right)$ holds. We now show that two signatures created by a single signer using a basename $\mathrm{bsn} \neq \perp$ can be linked. This is obvious from the description of the DAA scheme, as two signatures will have the same $(B, K)$ pair if the signatures are created using the same private key $f$.

Theorem 2 Under the $G_{1}-D D H$ assumption, the DAA scheme in Section 4 is user-controlled-anonymous. More specifically, if there is an adversary $\mathcal{A}$ that succeeds with a non-negligible probability to break the usercontrolled-anonymity game, then there is a polynomial-time algorithm $\mathcal{B}$ that solves the $G_{1}-D D H$ problem with a non-negligible probability.

Proof. Suppose an algorithm $\mathcal{A}$ breaks the user-controlled-anonymity game of the DAA scheme with nonnegligible probability. We can build a polynomial-time simulator $\mathcal{B}$ that breaks the $G_{1}$-DDH problem as follows. $\mathcal{B}$ is given as input a tuple $\left(u, v=u^{a}, w=u^{b}, z\right)$ where $u \leftarrow G_{1}, a, b, \leftarrow \mathbb{Z}_{p}$, and either $z=u^{a b}$ or $z$ is a random element in $G_{1}$. B decides which $z$ was given by interacting with $\mathcal{A}$ as follows.

We first give an overview of the proof. $\mathcal{B}$ first creates a special signer $S^{*}$ where its secret key $f=\log _{u} v$, however $\mathcal{B}$ does not know the secret key. $\mathcal{B}$ creates rest of the signers by running the join protocol with $\mathcal{A}$. To respond to a sign query for signer $S^{*}, \mathcal{B}$ simulates the signature using the $(u, v)$ pair. In the challenge phase, if $S^{*}$ is selected as one of the $\left(S_{0}, S_{1}\right)$ pair, $\mathcal{B}$ picks $S^{*}$ for creating a signature by simulating the signature using the $(w, z)$ pair, i.e., simulates using the secret key $f=\log _{w} z$. If $z=u^{a b}$, then $\log _{u} v=\log _{w} z, \mathcal{A}$ has non-negligible advantage guessing the random bit $b$ correctly. If $z \neq u^{a b}, \mathcal{A}$ does not have any advantage guessing $b$ or $\mathcal{A}$ may abort the game. $\mathcal{B}$ can use the output of $\mathcal{A}$ to decide whether $z=u^{a b}$.

Setup. Let $\left(G_{1}, G_{2}\right)$ be a bilinear group pair of prime order $p$ with generator $g_{1}$ and $g_{2}$, respectively. $\mathcal{B}$ chooses a random $\gamma \leftarrow \mathbb{Z}_{p}^{*}$ as isk and sets the public key gpk $=\left(G_{1}, G_{2}, G_{T}, p, e, g_{1}, h_{1}:=u, h_{2}, g_{2}, w:=\right.$ $\left.g_{2}^{\gamma}, H_{1}, H_{2}, H_{3}, H_{4}, H_{5}, T_{1}, T_{2}, T_{3}, T_{4}\right)$ by running the setup algorithm. $\mathcal{B}$ sends isk and gpk to $\mathcal{A}$.

Hash Queries. We model the hash functions $H_{2}, H_{3}$, and $H_{5}$ as three random oracles. $\mathcal{B}$ responds to the hash queries for $H_{2}, H_{3}$, and $H_{5}$ as follows.

- $H_{2}(m)$ : If $m$ has not been queried before, $\mathcal{B}$ chooses $H_{2}(m)$ uniformly at random from $\mathbb{Z}_{p}^{*}$ and returns it to $\mathcal{A}$, otherwise $\mathcal{B}$ returns the previously queried result on $m$ to ensure consistency. 
- $H_{3}(m)$ : Let $q_{h}$ be the expected number of unique $H_{3}$ queries. $\mathcal{B}$ chooses a random $i \leftarrow\left\{1, \ldots, q_{h}\right\}$. If $m$ has been queried before, $\mathcal{B}$ returns the previously queried result on $m$ to ensure consistency. Otherwise, if $m$ is the $i$-th unique query on $H_{3}, \mathcal{B}$ chooses chooses a random $r \leftarrow \mathbb{Z}_{p}^{*}$ and sets $H_{3}(m):=w^{r}$. For rest of the queries, $\mathcal{B}$ chooses a random $r \leftarrow \mathbb{Z}_{p}^{*}$ and sets $H_{3}(m):=u^{r}$. We use $\mathrm{bsn}^{*}$ to denote the $i$-th unique query.

- $H_{5}(m): \mathcal{B}$ chooses $H_{5}(m)$ uniformly at random from $\mathbb{Z}_{p}^{*}$ while ensuring consistency.

Join Queries. $\mathcal{A}$ requests for creating a new signer $S$. Let $q_{j}$ be the expected number of join requests from $\mathcal{A}$. $\mathcal{B}$ chooses a random $i \leftarrow\left\{1, \ldots, q_{j}\right\}$. There are two cases for $\mathcal{B}$ to respond:

- If the query is the $i$-th join query: $\mathcal{B}$ sets $F:=v$ without knowing the secret key $f=\log _{u} v$, and then forges rest of the join protocol as follows: it chooses randomly $c, s_{f} \leftarrow \mathbb{Z}_{p}$ and computes $R=h_{1}^{s_{f}} \cdot F^{-c}$. It then patches the oracle by setting $H_{2}\left(\operatorname{gpk}\left\|n_{I}\right\| F \| R\right):=c$. If $H_{2}\left(\operatorname{gpk}\left\|n_{I}\right\| F \| R\right)$ has been queried before, $\mathcal{B}$ quites and outputs "abortion 0". $\mathcal{B}$ receives a credential from $\mathcal{A}$. We use $S^{*}$ to denote the identity of this signer.

- If the query is not the $i$-th join query: $\mathcal{B}$ chooses a random $f \leftarrow \mathbb{Z}_{p}^{*}$, computes $F:=h_{1}^{f}$. If $F=v$, $\mathcal{B}$ quites and outputs "abortion 0". $\mathcal{B}$ runs the rest of the join protocol as the signer with $\mathcal{A}$ as the issuer, and obtains a credential $c r e=(A, x)$. $\mathcal{B}$ verifies cre and stores $(S, f, A, x)$ in its log.

Sign Queries. Given a signer's identity $S$, a message $m$ to be signed, a nonce $n_{V}$ from $\mathcal{A}$, a basename bsn, $\mathcal{B}$ responds with a signature $\sigma$ as follows: Assuming the signer $S$ has already joined, if $S$ is not $S^{*}, \mathcal{B}$ finds the corresponding secret key and credential $(f, A, x)$ associated with $S$, runs the sign protocol, and outputs $\sigma$ to $\mathcal{A}$. If $S=S^{*}, \mathcal{B}$ needs to forge a signature as follows:

1. If bsn $=\perp, \mathcal{B}$ chooses a random $r \leftarrow \mathbb{Z}_{p}$ and sets $B:=u^{r}$ and $K:=v^{r}$.

2. If $\mathrm{bsn}=\mathrm{bsn}^{*}, \mathcal{B}$ quits and outputs "abortion 1".

3. If $\mathrm{bsn} \neq\left\{\perp, \mathrm{bsn}^{*}\right\}, \mathcal{B}$ searches the $\log$ of $H_{3}$ queries and retrieves $r$ where $H_{3}(\mathrm{bsn})=u^{r}$. $\mathcal{B}$ sets $B:=u^{r}$ and computes $K:=v^{r}$.

4. $\mathcal{B}$ chooses $T \leftarrow G_{1}, n_{T} \leftarrow\{0,1\}^{t}$, and $c, s_{f}, s_{x}, s_{a}, s_{b} \leftarrow \mathbb{Z}_{p}$.

5. $\mathcal{B}$ computes $R_{1}:=B^{s_{f}} \cdot K^{-c}$.

6. $\mathcal{B}$ computes $R_{2}:=e\left(T, g_{2}\right)^{-s_{x}} \cdot e\left(h_{1}, g_{2}\right)^{s_{f}} \cdot e\left(h_{2}, g_{2}\right)^{s_{b}} \cdot e\left(h_{2}, w\right)^{s_{a}} \cdot\left(e\left(g_{1}, g_{2}\right) / e(T, w)\right)^{c}$.

7. $\mathcal{B}$ patches the oracle $H_{5}$ by setting $H_{5}\left(H_{4}\left(\operatorname{gpk}\|B\| K\|T\| R_{1}\left\|R_{2}\right\| n_{V}\right)\left\|n_{T}\right\| m\right):=c$. If $H 5\left(H_{4}(\operatorname{gpk} \|\right.$ $\left.\left.B\|K\| T\left\|R_{1}\right\| R_{2} \| n_{V}\right)\left\|n_{T}\right\| m\right)$ has been queried before, $\mathcal{B}$ quites and outputs "abortion 0".

8. $\mathcal{B}$ outputs the signature $\sigma:=\left(B, K, T, c, n_{T}, s_{f}, s_{x}, s_{a}, s_{b}\right)$.

Corrupt Queries. If a corrupt query is for a signer $S \neq S^{*}$, then $\mathcal{B}$ responds with the secret key corresponding to $S$. Otherwise, $\mathcal{B}$ quits and outputs "abortion 2".

Challenge. In the challenge, $\mathcal{A}$ outputs a message $m$, a basename bsn, and two signer's identity $S_{0}$ and $S_{1}$. If $S^{*} \notin\left\{S_{0}, S_{1}\right\}$ or bsn $\notin\{\perp$, bsn* $\}$, then $\mathcal{B}$ quits and outputs "abortion 3". Otherwise, $\mathcal{B}$ picks $b \in\{0,1\}$ such that $S_{b}=S^{*}$, and generates a signature $\sigma^{*}$ for $m$ by performing the following steps:

1. If bsn $=\perp, \mathcal{B}$ chooses a random $r \leftarrow \mathbb{Z}_{p}$ and sets $B:=w^{r}$ and $K:=z^{r}$.

2. If $\mathrm{bsn}=\mathrm{bsn}^{*}, \mathcal{B}$ searches the log of $H_{3}$ queries and retrieves $r$ where $H_{3}\left(\mathrm{bsn}^{*}\right)=w^{r}$. $\mathcal{B}$ sets $B:=w^{r}$ and computes $K:=z^{r}$.

3. The rest of the sign algorithm follows the sign queries above.

$\mathcal{B}$ sends the resulting $\sigma^{*}$ to $\mathcal{A}$.

Output. In the end, $\mathcal{A}$ outputs $b^{\prime} \in\{0,1\}$ as the guess for $b$ or aborts without any output. If $b=b^{\prime}$, then $\mathcal{B}$ outputs 1 , which means that $z=u^{a b}$. Otherwise $\mathcal{B}$ outputs 0 , which means that $z$ is a random element in $G_{1}$. 
We now discuss the probability that algorithm $\mathcal{B}$ does not abort in the above game. There are four cases where $\mathcal{B}$ can abort. We study each case as follows:

1. Abortion 0 . The chance of this type of abortion is $O(1 / p)$. Since $p$ is a large prime, the probability of this abortion is negligible.

2. Abortion 1. Recall that $\mathcal{A}$ cannot use the same non-empty bsn in the sign query and challenge query for signers $S_{0}$ and $S_{1}$. In other words, $\mathcal{A}$ cannot query all possible bsn for $S^{*}$ in the sign queries. The probability that $\mathcal{B}$ does not abort in this case is at least $1 / q_{h}$.

3. Abortion 2. As $\mathcal{A}$ cannot corrupt all the signers, the probability that $\mathcal{B}$ does not abort is at least $1 / q_{j}$.

4. Abortion 3. $\mathcal{B}$ does not abort in this case if $\mathcal{A}$ selects $S^{*}$ and $\mathrm{bsn}^{*}$ in the challenge query. Thus the probability that $\mathcal{B}$ does not abort in this case is $1 /\left(q_{h} \cdot q_{j}\right)$.

$\mathcal{B}$ does not abort if (1) bsn* was not chosen in the sign queries for $S^{*},(2) S^{*}$ was not chosen in the corrupt queries, and (3) $S^{*}$ and bsn* were chosen in the challenge query. The probability that $\mathcal{B}$ does not abort the above game is roughly $1 /\left(q_{h} \cdot q_{j}\right)$.

Let $\epsilon$ be the probability that $\mathcal{A}$ succeeds in breaking the user-controlled-anonymity game. Suppose $\mathcal{B}$ does not abort during the above simulation. If $z=u^{a b}$, then $\log _{u} v=\log _{w} z, \mathcal{B}$ simulates the game perfectly, i.e., $\operatorname{Pr}\left[b=b^{\prime}\right]>\frac{1}{2}+\epsilon$. If $z$ is a random element in $G_{1}$, then $\sigma^{*}$ in the challenge query is simulated using the $(w, z)$ pair. In other words, the secret key used in generated $\sigma^{*}$ is different from either secret key of $S_{0}$ or $S_{1}$. Observe that $\mathcal{B}$ in this case does not simulate the game perfectly, especially in the challenge query. $\mathcal{A}$ could abort the game. If $\mathcal{A}$ does not abort the game, $\mathcal{A}$ does not have any advantage guessing $b$. It follows that $\operatorname{Pr}\left[b=b^{\prime}\right]=\frac{1}{2}$. Therefore, assuming $\mathcal{B}$ does not abort, it has probability at least $\epsilon / 2$ in solving the DDH problem in $G_{1}$.

Theorem 3 Under the q-SDH assumption, the DAA scheme in Section 4 is user-controlled-traceable. More specifically, if there is an adversary $\mathcal{A}$ that succeeds with a non-negligible probability to break the usercontrolled-traceability game, then there is a polynomial-time algorithm $\mathcal{B}$ that solves the q-SDH problem with a non-negligible probability.

Proof. The proof of this theorem derives from the user-controlled-traceability proof in Chen DAA scheme [11]. We show how to construct an algorithm $\mathcal{B}$ that solves the $q$-SDH problem by interacting with $\mathcal{A}$ that succeeds with a non-negligible probability to break the user-controlled-traceability game. We use a technique from Boneh and Boyen [3] that, given $g_{1} \in G_{1}, g_{2} \in G_{2}, w=g_{2}^{\gamma}$, and $q-1 \mathrm{SDH}$ pairs $\left(B_{i}, x_{i}\right)$ such that $e\left(B_{i}, w g_{2}^{x_{i}}\right)=e\left(g_{1}, g_{2}\right)$, one more SDH pair $(B, x)$ can be transformed into a solution to the $q$-SDH problem. We now describe how $\mathcal{B}$ interacts with $\mathcal{A}$ as follows:

Setup. $\mathcal{B}$ runs the setup algorithm as follows: Let $e: G_{1} \times G_{2} \rightarrow G_{T}$ be a bilinear map function. $\mathcal{B}$ is given $\left(g_{1}, g_{2}, w\right)$ and $q-1 \mathrm{SDH}$ pairs $\left(B_{i}, x_{i}\right)$ as input, where $g_{1}=\psi\left(g_{2}\right), w=g_{2}^{\gamma}$, and $B_{i}=g_{1}^{1 /\left(\gamma+x_{i}\right)}$. $\mathcal{B}$ chooses $a, b, x \leftarrow \mathbb{Z}_{p}^{*}$ and computes

$$
h_{1}:=\psi\left(\left(\left(w \cdot g_{2}^{x}\right)^{b} \cdot g_{2}^{-1}\right)^{1 / a}\right)=g_{1}^{((\gamma+x) b-1) / a} .
$$

$\mathcal{B}$ sets the public key gpk $=\left(G_{1}, G_{2}, G_{T}, p, e, g_{1}, h_{1}, h_{2}, g_{2}, w, H_{1}, H_{2}, H_{3}, H_{4}, H_{5}, T_{1}, T_{2}, T_{3}, T_{4}\right)$. Note that $\mathcal{B}$ does not know the value of isk $=\gamma \cdot \mathcal{B}$ sends gpk to $\mathcal{A}$.

Hash Queries. We model the hash functions $H_{2}, H_{3}$, and $H_{5}$ as three random oracles. $\mathcal{B}$ responds to the hash queries for $H_{2}, H_{3}$, and $H_{5}$ as follows.

- $H_{2}(m): \mathcal{B}$ chooses $H_{2}(m)$ uniformly at random from $\mathbb{Z}_{p}^{*}$ while ensuring consistency.

- $H_{3}(m)$ : If $m$ has not been queried before, $\mathcal{B}$ chooses $H_{2}(m)$ uniformly at random from $G_{1}$ and returns it to $\mathcal{A}$, otherwise $\mathcal{B}$ returns the previously queried result on $m$ to ensure consistency.

- $H_{5}(m): \mathcal{B}$ chooses $H_{5}(m)$ uniformly at random from $\mathbb{Z}_{p}^{*}$ while ensuring consistency. 
Join Queries. $\mathcal{A}$ requests for creating a new signer $S$. There are two cases of the join queries as defined by the user-controlled-traceability game in Section 2 :

1. Given a new signer $S, \mathcal{B}$ has freedom to choose sk of its choice and then creates cre.

2. Given a new signer $S$ and a secret key sk chosen by the $\mathcal{A}, \mathcal{B}$ creates a credential cre for sk. $\mathcal{B}$ sends cre to $\mathcal{A}$ and put sk in the revocation list RL.

For case $1, \mathcal{B}$ selects one query randomly and sets $f:=a$ and cre $:=(A, x)$ where $A:=g_{1}^{b}$. For rest of the queries, either $\mathcal{B}$ chooses $f_{i}$ at random (case 1 ) or receives $f_{i}$ from $\mathcal{A}, \mathcal{B}$ uses one $\mathrm{SDH}$ pair $\left(B_{i}, x_{i}\right)$ to computes $\operatorname{cre}_{i}:=\left(A_{i}, x_{i}\right)$ for $f_{i}$ as follows:

$$
A_{i}=\left(g_{1} \cdot h_{1}^{f_{i}}\right)^{1 /\left(\gamma+x_{i}\right)}=B_{i}^{1-f_{i} / a+f_{i} b(x+\gamma) / a}:=B_{i}^{1-f_{i} / a+f_{i} b\left(x-x_{i}\right) / a} \cdot g_{1}^{f_{i} b / a} .
$$

Corrupt Queries. $\mathcal{A}$ requests to corrupt a signer $S$. We assume that the signer $S$ has been joined before, $\mathcal{B}$ responds with the secret key sk and credential cre corresponding to $S$. $\mathcal{B}$ inserts sk to the revocation list RL.

Sign Queries. Given a signer's identity $S$, a message $m$ to be signed, a nonce $n_{V}$ from $\mathcal{A}$, a basename bsn, $\mathcal{B}$ computes the signature $\sigma$ by invoking the sign protocol. $\mathcal{B}$ returns $\sigma$ to $\mathcal{A}$.

Semi-sign Queries. Given a signer's identity $S$, a message $m$ to be signed, a nonce $n_{V}$ from $\mathcal{A}$, a basename bsn, $\mathcal{B}$ runs the sign protocol as a TPM by interacting with $\mathcal{A}$ as a host. $\mathcal{B}$ computes $B$, chooses $r_{f}$ at random, computes $K, R_{1}, R_{2 t}$, and sends $\left(B, K, R_{1}, R_{2 t}\right)$ to $\mathcal{A}$. On receiving $c_{h}$ from $\mathcal{A}, \mathcal{B}$ chooses $n_{T}$ at random and computes $c$ and $s_{f}$.

Output. In the end, $\mathcal{A}$ outputs a signer $S$, a message $m$, a basename bsn, and a signature $\sigma$. There are two cases to consider:

1. If $\sigma$ can be successfully verified, i.e., $\operatorname{Verify}(m$, bsn, $\sigma$, gpk, RL $)=1, \mathcal{B}$ can rewind $\mathcal{A}$ to extract the underlying the signing key $\left(f^{*}, A^{*}, x^{*}\right)$ such that $e\left(A^{*}, w g_{2}^{x^{*}}\right)=e\left(g_{1} h_{1}^{f^{*}}, g_{2}\right)$. To rewind $\mathcal{A}, \mathcal{B}$ controls the result of $H_{5}$ by choosing two different $c$ values $c^{\prime}$ and $c^{\prime \prime}$ to the same $B, K, T, R_{1}, R_{2}$. $\mathcal{A}$ responds with $s_{f}^{\prime}, s_{x}^{\prime}, s_{a}^{\prime}, s_{b}^{\prime}$ and $s_{f}^{\prime \prime}, s_{x}^{\prime \prime}, s_{a}^{\prime \prime}, s_{b}^{\prime \prime}$. $\mathcal{B}$ computes $\Delta=c^{\prime}-c^{\prime \prime}$ and extracts

$$
f^{*}:=\left(s_{f}^{\prime}-s_{f}^{\prime \prime}\right) / \Delta, \quad x^{*}:=\left(s_{x}^{\prime}-s_{x}^{\prime \prime}\right) / \Delta, \quad a^{*}:=\left(s_{a}^{\prime}-s_{a}^{\prime \prime}\right) / \Delta, \quad A^{*}:=T /\left(h_{2}^{a^{*}}\right) .
$$

Also note that $f^{*} \notin \mathrm{RL}$, otherwise, signature verification would fail. There are two possible results (a) If $x^{*} \notin\left\{x_{i}, x\right\}$ for any $i, \mathcal{B}$ can compute

$$
B^{*}:=\left(A^{*} \cdot g_{1}^{-b f^{*} / a}\right)^{a /\left(a-f^{*}+b f^{*}\left(x-x^{*}\right)\right)} .
$$

(b) If $x^{*} \in\left\{x_{i}, x\right\}$ and $A^{*} \notin\left\{A_{i}, A\right\}$ for any $i$. If $e^{*} \neq e, \mathcal{B}$ aborts and outputs failure. With the probability of $1 / q, e^{*}=e, \mathcal{B}$ computes

$$
B^{*}:=\left(A^{*} \cdot g_{1}^{-b f^{*} / a}\right)^{a /\left(a-f^{*}\right)} .
$$

In either of the above two cases, $\mathcal{B}$ obtains $\left(B^{*}, x^{*}\right)$ as the extra SDH pair so that it can solve the given $q$-SDH problem.

2. If $\mathrm{bsn} \neq \perp, \mathcal{A}$ wins in this case if there exists another signature $\sigma^{\prime}$ by the signer $S$ using the same basename bsn from the sign queries, such that $\operatorname{Link}\left(\sigma, \sigma^{\prime}, \mathrm{gpk}\right)=0$. Let $\sigma=\left(B, K, T, c, n_{T}, s_{f}, s_{x}\right.$, $\left.s_{a}, s_{b}\right)$ and $\sigma^{\prime}=\left(B^{\prime}, K^{\prime}, T^{\prime}, c^{\prime}, n_{T}^{\prime}, s_{f}^{\prime}, s_{x}^{\prime}, s_{a}^{\prime}, s_{b}^{\prime}\right)$. Since the same bsn is used, $B=B^{\prime}$. Because two signatures are unlinkable, thus $K \neq K^{\prime}$. This means $\mathcal{A}$ managed to create a different sk for the signer $S . \mathcal{B}$ can use the same method as case (1) to extract a different $(f, A, x)$ tuple as a valid secret key and credential pair, thus obtain an extra SDH pair. $\mathcal{B}$ can also solve the $q$-SDH problem in this case.

In either of the above two cases, $\mathcal{B}$ can solve the $q$-SDH problem with a non-negligible probability if $\mathcal{A}$ can wins the game with a non-negligible probability. 


\section{Comparisons with Existing DAA Schemes}

In this section, we compare our DAA scheme with several existing pairing-based DAA schemes $[6,13,15,11]$. Note that we do not include the original CMS-DAA scheme [12] in the comparisons, as it is not secure, instead we compare ours with the patched version [13]. We also do not include the pairing-based EPID scheme [9] in the comparison, because EPID does not have the feature of splitting computation between a TPM and a host.

We compare the credential and signature sizes of our scheme with other DAA schemes in Table 1 . We use $\mathbb{Z}_{q}$ to denote the size of an element in $\mathbb{Z}_{q}, h$ to denote the size of a hash result, $G_{1}$ to denote the size of an element in $G_{1}$, and $G_{T}$ to denote the size of an element in $G_{T}$. For bilinear maps with 128-bit security, $G_{T}$ needs to be around 3072 bits [17]. Our DAA scheme has the same credential and signature sizes as in Chen's DAA scheme [11]. The credential and signature sizes in our scheme are smaller than other DAA schemes $[6,13,15]$.

\begin{tabular}{|c|c|c|}
\hline DAA Scheme & Credential Size & Signature Size \\
\hline Scheme of [6] & $3 G_{1}$ & $2 \mathbb{Z}_{q}+3 G_{1}+2 G_{T}+1 h$ \\
\hline Scheme of [13] & $3 G_{1}$ & $1 \mathbb{Z}_{q}+5 G_{1}+1 h$ \\
\hline Scheme of [15] & $2 \mathbb{Z}_{q}+1 G_{1}$ & $6 \mathbb{Z}_{q}+2 G_{1}+2 G_{T}+1 h$ \\
\hline Scheme of [1] & $1 \mathbb{Z}_{q}+1 G_{1}$ & $4 \mathbb{Z}_{q}+3 G_{1}+1 h$ \\
\hline Our Scheme & $1 \mathbb{Z}_{q}+1 G_{1}$ & $4 \mathbb{Z}_{q}+3 G_{1}+1 h$ \\
\hline
\end{tabular}

Table 1: A comparison between our DAA scheme and other DAA schemes in credential and signature sizes

We compare the efficiency of signing and verification algorithms of our scheme with other DAA schemes in Table 2. We use $P$ to denote a pairing operation, $G_{1}$ to denote an exponentiation operation in $G_{1}, G_{1}^{2}$ to denote a multi-exponentiation operation, and so on. A multi-exponentiation is slightly more expensive than an exponentiation. As we mentioned earlier in Section 1, operations in $G_{1}$ are much more efficient than ones in $G_{T}$. Therefore, our DAA scheme has significant advantage for computationally weak device such as TPM. The efficiency of the sign protocol for our scheme is approximately 5 times more efficient than the rest of pairing-based DAA schemes $[6,13,15,11]$ using Barreto-Naehrig curves.

\begin{tabular}{|c|c|c|c|}
\hline DAA Scheme & TPM Sign & Host Sign & Verify \\
\hline Scheme of [7] & $3 G_{T}$ & $3 G_{1}+1 G_{T}+3 P$ & $1 G_{T}^{2}+1 G_{T}^{3}+5 P+(n+1) G_{T}$ \\
\hline Scheme of [13] & $2 G_{1}+1 G_{T}$ & $3 G_{1}+1 P$ & $1 G_{T}^{2}+1 G_{T}^{2}+5 P+n G_{1}$ \\
\hline Scheme of $[15]$ & $2 G_{1}+1 G_{T}^{2}$ & $1 G_{1}+2 G_{1}^{2}+1 G_{1}^{3}+1 G_{T}^{3}$ & $1 G_{1}^{2}+2 G_{1}^{3}+1 G_{T}^{5}+3 P+n G_{T}$ \\
\hline Scheme of $[11]$ & $2 G_{1}+1 G_{T}$ & $1 G_{1}+1 G_{T}^{3}$ & $1 G_{1}^{2}+1 G_{2}^{2}+1 G_{T}^{4}+1 P+n G_{1}$ \\
\hline Our Scheme & $3 G_{1}$ & $1 G_{1}+1 G_{1}^{2}+1 G_{T}+1 P$ & $1 G_{1}^{2}+1 G_{2}^{2}+1 G_{T}^{4}+1 P+n G_{1}$ \\
\hline
\end{tabular}

Table 2: A comparison between our DAA scheme and other DAA schemes in the efficiency of sign and verify algorithms

Observe that the efficiency we gain in TPM comes with a price - the host needs to perform an additional pairing. The total efficiency of the sign protocol is $4 G_{1}+1 G_{1}^{2}+1 G_{T}+1 P$ in our scheme, whereas it is $3 G_{1}+1 G_{T}+3 G_{T}^{3}$ in Chen's DAA scheme [11]. Note that TPM is much slower than the host platform, e.g., probably 100 times slower or more. Based on the performance simulation by Chen, Page, and Smart [14], a pairing operation on a host takes less than 15 millie-seconds for a 64 -bit $2.4 \mathrm{GHz}$ Intel Core 2 processor, while $2 G_{1}+1 G_{T}$ operation takes more than 3 seconds for a 32 -bit $33 \mathrm{MHz}$ simulated TPM. Base on these performance data, we estimate that the overall sign protocol will take less than 1 second in our scheme, whereas the sign protocol in $[11,13]$ will take more than 3 seconds.

We did not compare the efficiency of the join protocol because the join protocol is executed much less frequently than the sign protocol or the verification algorithm. Besides the join protocol in our DAA scheme is the same as the one in Chen's DAA scheme [11], thus has the same efficiency. 


\section{Conclusions}

In this paper, we proposed an efficient DAA scheme from bilinear maps. Our new DAA scheme takes much less resources for TPM implementation (both computation and software code size) compared to the existing DAA schemes. We believe our DAA scheme is a good candidate for the next generation of TPM initiated by the TCG TPM working group, assuming that a TPM can support multiple DAA algorithms.

\section{References}

[1] Michael Backes, Matteo Maffei, and Dominique Unruh. Zero-knowledge in the applied pi-calculus and automated verification of the direct anonymous attestation protocol. In Proceedings of IEEE Symposium on Security and Privacy, pages 202-215. IEEE Computer Society, 2008.

[2] Paulo S. L. M. Barreto and Michael Naehrig. Pairing-friendly elliptic curves of prime order. In Proceedings of the 12th International Workshop on Selected Areas in Cryptography, volume 3897 of LNCS, pages 319-331. Springer, 2005.

[3] Dan Boneh and Xavier Boyen. Short signatures without random oracles. In Advances in Cryptology EUROCRYPT '04, volume 3027 of LNCS, pages 56-73. Springer, 2004.

[4] Dan Boneh, Xavier Boyen, and Hovav Shacham. Short group signatures. In Advances in Cryptology CRYPTO '04, volume 3152 of LNCS, pages 41-55. Springer, 2004.

[5] Ernie Brickell, Jan Camenisch, and Liqun Chen. Direct anonymous attestation. In Proceedings of the 11th ACM Conference on Computer and Communications Security, pages 132-145. ACM Press, 2004.

[6] Ernie Brickell, Liqun Chen, and Jiangtao Li. A new direct anonymous attestation scheme from bilinear maps. In Proceedings of 1st International Conference on Trusted Computing, volume 4968 of LNCS, pages 166-178. Springer, 2008.

[7] Ernie Brickell, Liqun Chen, and Jiangtao Li. Simplified security notions of direct anonymous attestation and a concrete scheme from pairings. International Journal of Information Security, 8(5):315-330, 2009.

[8] Ernie Brickell and Jiangtao Li. Enhanced Privacy ID: A direct anonymous attestation scheme with enhanced revocation capabilities. In Proceedings of the 6th ACM Workshop on Privacy in the Electronic Society, pages 21-30. ACM Press, October 2007.

[9] Ernie Brickell and Jiangtao Li. Enhanced Privacy ID from bilinear pairing. Cryptology ePrint Archive, Report 2009/095, 2009. http://eprint.iacr.org/.

[10] Jan Camenisch and Jens Groth. Group signatures: Better efficiency and new theoretical aspects. In Proceedings of 4th International Conference on Security in Communication Networks, volume 3352 of LNCS, pages 122-135. Springer, 2005.

[11] Liqun Chen. A DAA scheme requiring less TPM resources. In Proceedings of the 5th China International Conference on Information Security and Cryptology, LNCS. Springer, 2009.

[12] Liqun Chen, Paul Morrissey, and Nigel P. Smart. Pairings in trusted computing. In Proceedings of the 2nd Internation Conference on Pairing-Based Cryptography, volume 5209 of LNCS, pages 1-17. Springer, 2008.

[13] Liqun Chen, Paul Morrissey, and Nigel P. Smart. DAA: Fixing the pairing based protocols. Cryptology ePrint Archive, Report 2009/198, 2009. http://eprint.iacr.org/.

[14] Liqun Chen, Dan Page, and Nigel P. Smart. On the design and implementation of an efficient DAA scheme. Cryptology ePrint Archive, Report 2009/598, 2009. http://eprint.iacr.org/.

[15] Xiaofeng Chen and Dengguo Feng. Direct anonymous attestation for next generation tpm. Journal of Computers, 3(12):43-50, 2008. 
[16] Jung Hee Cheon. Security analysis of the strong diffie-hellman problem. In Advances in Cryptology EUROCRYPT' '06, volume 4004 of LNCS, pages 1-11. Springer, 2006.

[17] Neal Koblitz and Alfred Menezes. Pairing-based cryptography at high security levels. In Proceedings of the 10th IMA International Conference on Cryptography and Coding, volume 3796 of LNCS, pages 13-36. Springer, 2005.

[18] Adrian Leung and Chris J. Mitchell. Ninja: Non identity based, privacy preserving authentication for ubiquitous environments. In Proceedings of 9th International Conference on Ubiquitous Computing, volume 4717 of $L N C S$, pages 73-90. Springer, 2007.

[19] Anna Lysyanskaya, Ronald L. Rivest, Amit Sahai, and Stefan Wolf. Pseudonym systems. In Proceedings of the 6th Workshop on Selected Areas in Cryptography, volume 1758 of LNCS, pages 184-199. Springer, 1999.

[20] Victor Shoup. Lower bounds for discrete logarithms and related problems. In Advances in Cryptology - EUROCRYPT '97, volume 1233 of LNCS, pages 256-266. Springer, 1997.

[21] Trusted Computing Group. TCG TPM specification 1.2, 2003. Available at http://www. trustedcomputinggroup.org.

[22] Trusted Computing Group website. http://www.trustedcomputinggroup.org. 